\title{
Spontaneous bilateral internal carotid and vertebral artery dissections with dominant-hemisphere circulation maintained by external carotid artery-ophthalmic artery anastomoses
}

\author{
Danielle Golub, BA, ${ }^{1,4}$ Lizbeth Hu, BS, ${ }^{2}$ Siddhant Dogra, BS, ${ }^{3}$ Jose Torres, MD, ${ }^{4}$ and \\ Maksim Shapiro, MD ${ }^{3,4}$
} ${ }^{1}$ Department of Neurosurgery, ${ }^{2}$ Department of Otolaryngology, ${ }^{3}$ Department of Radiology, and ${ }^{4}$ Department of Neurology, New
York University School of Medicine, New York, New York

\begin{abstract}
Spontaneous cervical artery dissection (sCAD) is a major cause of stroke in young adults. Multiple sCAD is a rarer, more poorly understood presentation of SCAD that has been increasingly attributed to cervical trauma such as spinal manipulation or genetic polymorphisms in extracellular matrix components. The authors present the case of a 49-yearold, otherwise healthy woman, who over the course of 2 weeks developed progressive, hemodynamically significant, bilateral internal carotid artery and vertebral artery dissections. Collateral response involved extensive external carotid artery-internal carotid artery anastomoses via the ophthalmic artery, which were instrumental in maintaining perfusion because circle of Willis and leptomeningeal anastomotic responses were hampered by the dissection burden in the corresponding collateral vessels. Endovascular intervention by placement of Pipeline embolization devices and Atlas stents in bilateral internal carotid arteries was successfully performed. No syndromic or systemic etiology was discovered during a thorough workup.
\end{abstract} https://thejns.org/doi/abs/10.3171/2018.11.FOCUS18443

KEYWORDS spontaneous cervical artery dissection; carotid dissection; vertebral dissection; flow diverter; meningolacrimal artery

$\mathrm{S}$ PONTANEOUS cervical artery dissection (sCAD) is an important cause of stroke in young and middle-aged adults. The mean age at presentation is $45-48$ years old and there is no gender predilection. ${ }^{26,31,37}$ Dissection comprises $4 \%-5 \%$ of all ischemic strokes and is estimated to be the etiology for $25 \%$ of strokes in young or middleaged patients. Cervical artery dissections typically result from subintimal tears, leading to the establishment of a false lumen in the vessel wall. This can lead to a variety of untoward events, including narrowing or occlusion of the true lumen with secondary distal hypoperfusion, thromboembolism, vessel rupture, and delayed pseudoaneurysm formation. ${ }^{11,26,31,37}$

Mechanical stress to the arterial wall after trauma, cervical spine manipulation, or long-standing hypertension can provoke dissection. ${ }^{11,36}$ However, underlying arteriopathy or connective tissue defects are probably ultimately responsible for many sCAD cases.,11-13,36 Interestingly, patients with monogenic connective tissue disorders including Ehlers-Danlos syndrome, Marfan syndrome, and osteogenesis imperfecta represent the minority $(5 \%)$ of sCAD patients. ${ }^{13,37}$ Skin biopsies have shown that patients with nonsyndromic sCAD also harbor abnormalities in the ultrastructure of connective tissues. ${ }^{5,6,43}$ Genomic sequencing data from nonsyndromic patients have suggested single-nucleotide polymorphisms in candidate genes such as MTHFR, LOXL1, and PHACTR 1.,10,20,23,25

sCAD presents with a variety of local or regional pain syndromes and/or various neurological deficits depending on site of dissection, mechanism of ischemia (occlusive vs embolic), and availability of collateral vessels.,11,37,38 In this report, we describe the unique presentation, emergency endovascular management, and vascular anatomy of a woman with multivessel sCAD and preserved neurologi-

ABBREVIATIONS CCA = common carotid artery; CTA = CT angiography; ECA = external carotid artery; ICA = internal carotid artery; MCA = middle cerebral artery; MMA = middle meningeal artery; $M R A=M R$ angiography; NIHSS = National Institutes of Health Stroke Scale; OphA = ophthalmic artery; PCA = posterior cerebral artery; PCoA = posterior communicating artery; PED = Pipeline embolization device; sCAD = spontaneous cervical artery dissection; VA = vertebral artery.

SUBMITTED August 22, 2018. ACCEPTED November 8, 2018.

INCLUDE WHEN CITING DOI: 10.3171/2018.11.FOCUS18443. 


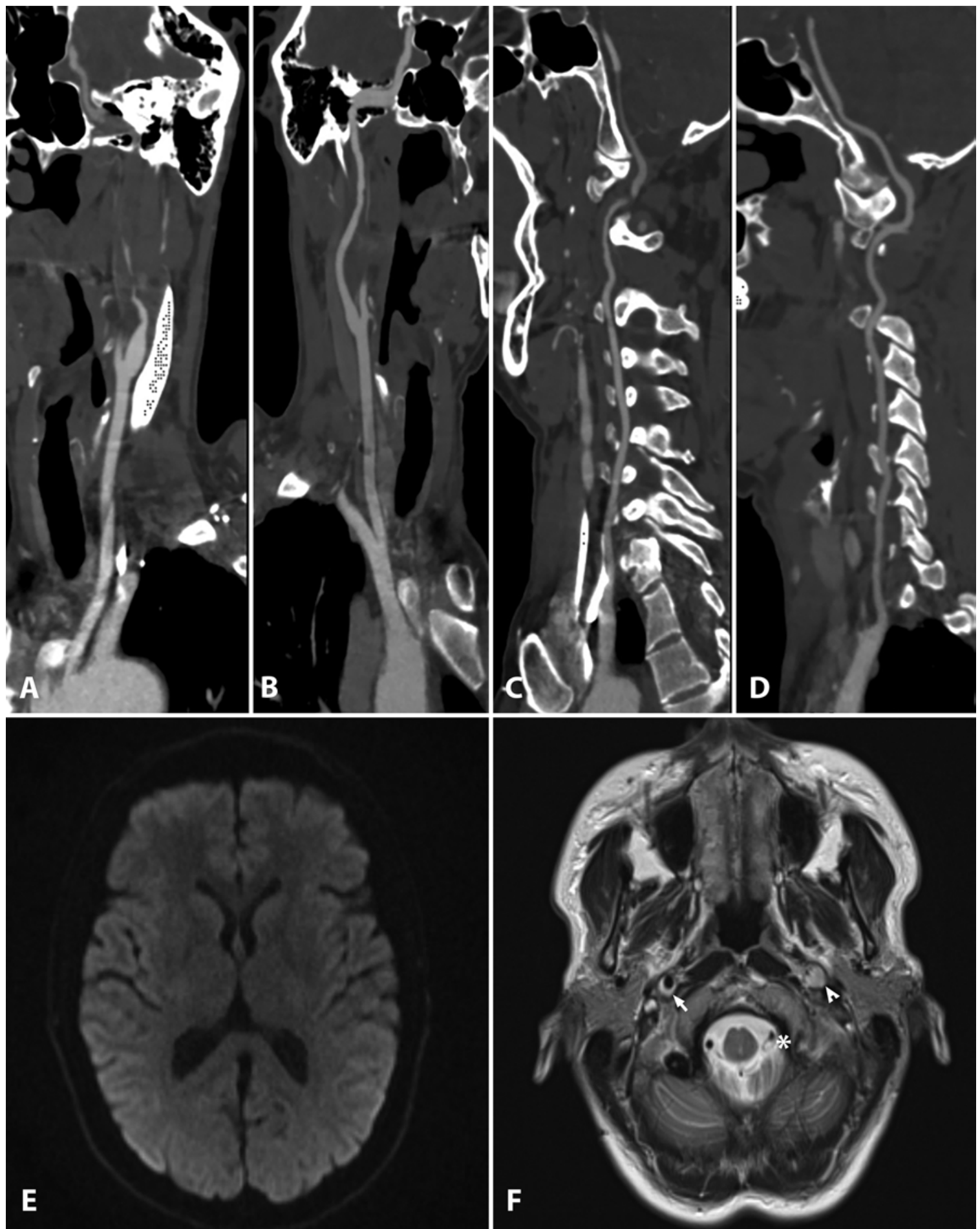

FIG. 1. A-D: 3D centerline reconstructions of cervical vessels on admission CT angiogram. Left ICA (A) showing critical stenosis starting distal to the carotid bulb through to the petrous segment and persistent irregularity in the cavernous segment; right ICA (B) showing irregular vessel caliber at the cervical segment, but with good contrast filling; left VA (C) showing a critical stenosis at the $V_{3}$ segment; right VA (D) with no abnormality. E: Diffusion-weighted MRI sequence obtained on admission showing no diffusion restriction or evidence of ischemia. F: T2-weighted MRI showing multivessel simultaneous dissection as diminished caliber of flow voids accompanied by surrounding intramural hematoma in the right ICA (arrow), left ICA (arrowhead), and left VA (asterisk). 
cal function attributable to a robust congenital anastomosis between the external carotid artery (ECA) and ophthalmic artery (OphA) systems.

\section{Case Report}

History

A 49-year-old woman presented to the emergency department with 3 hours of mild right-hand weakness and mild headache. She had a history of migraine and a remote history of C6-7 anterior cervical discectomy and fusion. She intermittently participated in hot yoga and Pilates, but denied recent trauma, chiropractic manipulations, or family history of stroke or vasculopathy. She insisted that her headache did not resemble her typical migraines, nor had she ever had migraines with auras. She had reported similar symptoms 5 days prior; CT angiography (CTA) performed at another institution was suggestive of a left internal carotid artery (ICA) dissection, for which she was medically managed with aspirin and clopidogrel.

\section{Examination and Initial Workup}

When the patient was initially triaged, a stroke code was called. Examination revealed only a slight slowing in rapid right-hand finger movements (National Institutes of Health Stroke Scale [NIHSS] score $=0$ ). Emergency head CT scans obtained without contrast showed no acute intracranial pathology. However, given the mild asymmetry in her finger movements in the context of mild headache and recent history, vascular imaging was pursued.

Admission CTA of the head and neck revealed abrupt tapering of the proximal left ICA at the level of the C3 vertebral body, followed distally by a severe $>90 \%$ stenosis with persistent irregularity through the cavernous segment-consistent with severe dissection (Fig. 1A). Also noted was new irregular appearance of the right ICA at the cervical segment with approximately $50 \%$ stenosis (Fig. 1B). Additionally present was a new critical stenosis in the left vertebral artery (VA) at the $\mathrm{V}_{3}$ segment (Fig. 1C). The right VA was unremarkable (Fig. 1D). The aortic arch and origins of the great vessels were normal.

MRI was obtained without contrast to rule out infarction, and showed no acute ischemia. Again seen were dissections of the bilateral ICAs and left VA on T2-weighted imaging (Fig. 1E and F).

\section{Management}

The patient was admitted to the stroke unit. She was medically managed with aspirin, clopidogrel, and atorvastatin and closely monitored. Given the critical nature and unclear etiology for her multiple sCADs, she consented to an urgent diagnostic angiogram with a possibility for intervention.

\section{Endovascular Procedure and Findings}

Under general anesthesia, right femoral artery access was obtained in the usual fashion via a 5-Fr catheter. Initial imaging of the descending aorta, renal vasculature, aortic arch, and proximal great vessels showed no abnormality. Digital subtraction angiography of the right VA showed leptomeningeal anastomoses between the right posterior

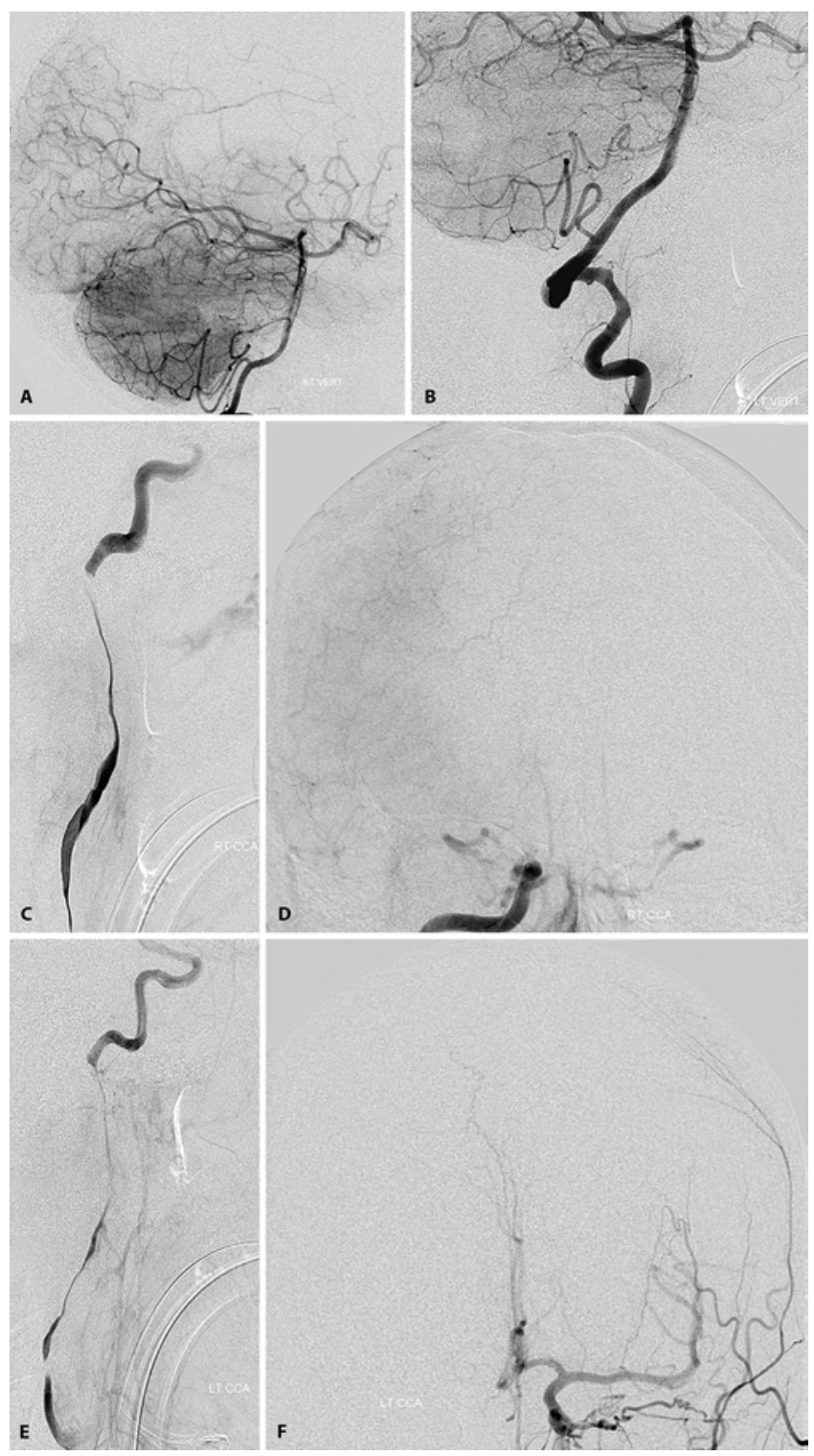

FIG. 2. Diagnostic angiograms of cervical vessels obtained on second day of admission. Right VA injection demonstrating extensive leptomeningeal MCA-PCA anastomoses and retrograde flow through a fetal $\mathrm{PCoA}$ in the presence of a hemodynamic stress from the stenosis in the anterior circulation (A). PCoA also visualized in the injection of the left VA (B). Acute right ICA critical stenosis seen on right CCA injection (C) with corresponding loss of perfusion of the right hemisphere (D). Known left ICA critical stenosis seen on left CCA injection (E) with distal perfusion preserved, probably from retrograde collateral flow, given the early appearance of an ECA-ICA anastomosis between the MMA and OphA (F).

cerebral artery (PCA) and middle cerebral artery (MCA) and retrograde flow through a fetal posterior communicating artery $(\mathrm{PCoA})$ to the anterior circulation-suggestive of an acute anterior circulation flow constraint (Fig. $2 \mathrm{~A}$ and $\mathrm{B}$ ). Right common carotid artery (CCA) imaging revealed severe dissection with near-complete occlusion of the distal cervical ICA segment-a marked overnight progression from the most recent CTA or MRI sequences (Fig. 2C and D). Left CCA imaging again demonstrated 

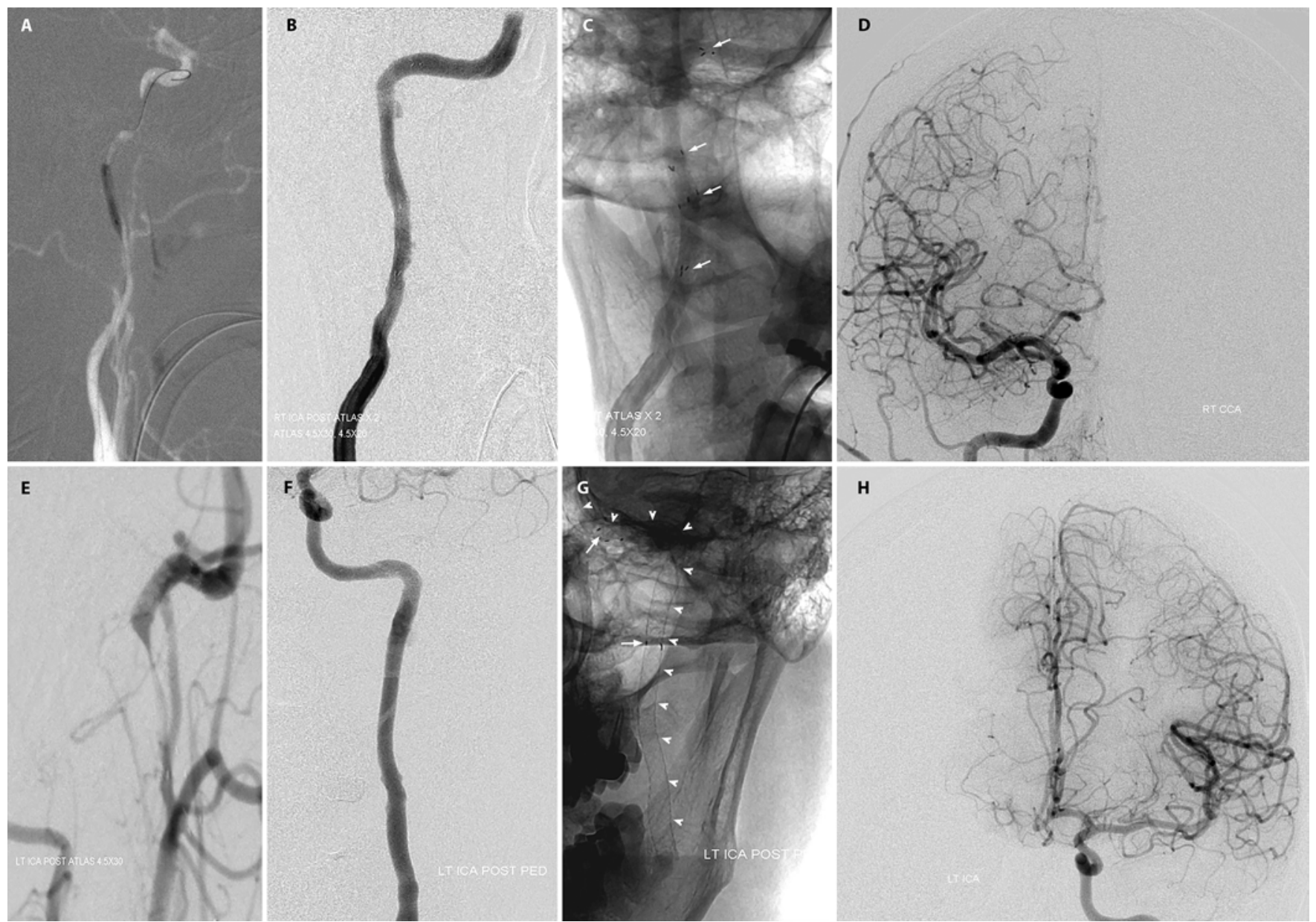

FIG. 3. A-D: Emergency intervention for revascularization of acute right ICA stenosis. Maverick balloon angioplasty of distal and middle segments of the right ICA (A). Restored ICA vessel caliber after balloon angioplasty and placement of 2 Atlas stents (B). Placement of Atlas stents in cervical and petrous segments of the right ICA visualized by radiopaque ends (arrows) (C). Postintervention right ICA injection showing restoration of right hemispheric perfusion (D). E-H: Intervention for revascularization of critical left ICA stenosis. Failure of first Atlas stent to restore vessel caliber $(E)$. Restoration of left ICA vessel caliber after additional placement of 2 PEDs (F). PED span (arrowheads) from cervical to cavernous segments of the left ICA visualized with radiopaque ends of anchoring Atlas stent (arrows) seen in petrous and distal cervical segments (G). Postintervention left ICA injection showing adequate left hemispheric perfusion $(\mathrm{H})$.

critical stenosis of distal left ICA (Fig. 2E). Cranial views showed early retrograde flow through the left OphA from the ECA circulation reconstituting the left ICA territory (Fig. 2F). Irregular vessel caliber with a 30\% stenosis was seen in the left VA at the level of C1 (Fig. 2B).

Given rapid progression of the right ICA dissection and after discussion with the patient's husband, himself a physician, emergency revascularization was undertaken. Balloon angioplasty of distal and middle segments of the right ICA was performed using a Maverick $4 \times 15-\mathrm{mm}$ balloon, with partial restoration of vessel caliber (Fig. 3A). Two Atlas stents (Stryker) measuring $4.5 \times 30 \mathrm{~mm}$ and 4.5 $\times 20 \mathrm{~mm}$ were sequentially deployed from the horizontal petrous to midcervical segments, restoring full luminal patency and cerebral perfusion (Fig. 3B-D).

The left CCA was then similarly catheterized, followed by placement of a $4.5 \times 30-\mathrm{mm}$ Atlas stent from the horizontal petrous to upper cervical segments of the left ICA with minimal improvement in vessel patency (Fig. 3E). Balloon angioplasty was then performed, followed by the sequential deployment of two $5 \times 25-\mathrm{mm}$ Pipeline embolization devices (PEDs) spanning the vertical cavernous segment to the proximal cervical segment (Fig. 3F and G). Restoration of luminal caliber and normalization of cerebral perfusion were observed (Fig. 3F and H). The left VA dissection did not have hemodynamic significance and was not treated.

After hemostasis was achieved, the patient emerged from anesthesia without neurological deficits. Right-hand rapid finger movements were restored to baseline. She was discharged to the stroke unit in stable condition.

\section{Postoperative Course}

Postintervention CTA showed improved flow through both anterior and posterior circulations despite persistent intramural hematomas in the cervical segments of both 
ICAs and the left VA. Other workup excluded inflammatory, autoimmune, or infectious vasculitis and hyperhomocysteinemia, and fibromuscular dysplasia was unlikely given normal results on the aortogram. A genetic polymorphism combined with increased risk from her history of anterior cervical discectomy and fusion was the most likely mechanism for her 3-vessel sCAD ${ }^{28}$ She was discharged with an NIHSS score of 0 on hospital day 3 . Close follow-up imaging 2 weeks later showed asymptomatic, persistent, irregular narrowing of the midcervical right ICA, which was treated by placement of a single $5 \times$ 25-mm PED to cover the previously placed Atlas stents and the residual narrowed segment (Fig. 4A and B). Also found was yet another new, mild, right VA dissection that was managed conservatively.

MR angiography (MRA) sequences obtained at 3 and 6 months showed progressive healing of all dissections with only mild residual stenosis of the distal cervical left ICA (Fig. 4C and D). The patient remains without neurological deficit and is being maintained on dual-antiplatelet therapy given the dissection burden and lengths of the stented segments. A lifelong ban on cervical neck stress, including yoga, was advised.

\section{Discussion}

Multiple sCAD is a rare, life-threatening pathology for which the etiology is frequently unknown. Approximately $20 \%$ of patients with sCAD have multivessel involvement, but only a handful of these cases are explained by syndromic connective tissue disorders. ${ }^{21}$ The diagnosis of multivessel sCAD continues to be made most reliably by catheter angiography because MRA and CTA have relatively worse sensitivity. The elusiveness of this diagnosis is exaggerated by its wildly variable presentation. ${ }^{21,41}$ Treating these patients is further complicated by the inherentprobably genetic-instability of the involved vasculature, compounded by the plurality of the insult.

The goals in managing dissection are to prevent progression, limit thromboembolic events, and preserve distal organ perfusion. Stabilizing the dissection and preventing the formation of thrombus with antiplatelet, anticoagulation, or antifibrinolytic agents is routine. The recent CADISS (Cervical Artery Dissection in Stroke Study) trial suggested that antiplatelet and anticoagulant drugs have equal efficacy in preventing stroke or death in cervical dissections, but intracranial hemorrhage was more likely with anticoagulants. ${ }^{30}$ In cases with intracranial extension, a high rate of associated subarachnoid hemorrhage has been observed, making dual-antiplatelet therapy a better choice for our patient over intravenous fibrinolytics or anticoagulation. ${ }^{9,42}$ In critical cases, endovascular intervention is highly favored over open surgical repair due to its exceedingly lower perioperative stroke risk $(0.02 \%$ vs $10 \%)$ and mortality rate (nearly $0 \%$ vs $2 \%) .33,45$ Our patient's critical stenoses made her a candidate for acute endovascular intervention especially since she, a young, active woman, was experiencing recurring dominant-hemisphere symptoms at rest (despite collateral circulation), putting her at high risk for future hypoperfusion and possible stroke., ${ }^{3,16}$

The right ICA stenosis was prioritized given the over-

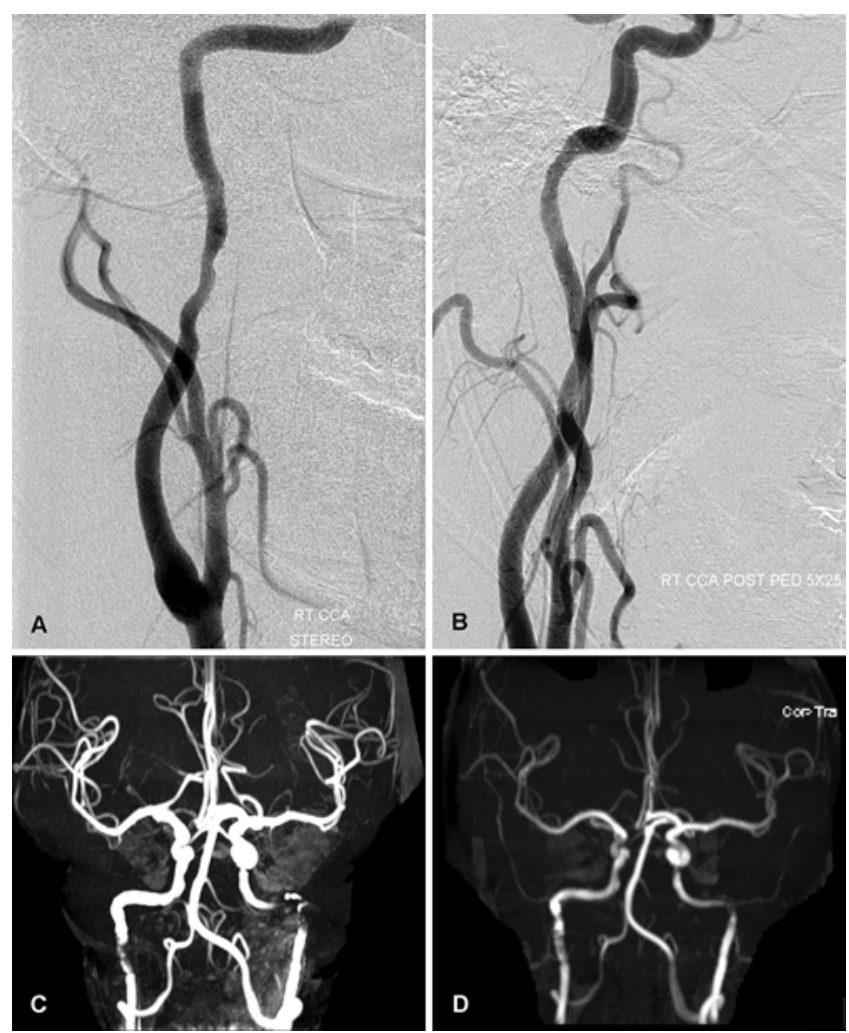

FIG. 4. A and B: Catheterization of the right ICA showing stable position of Atlas stents spanning the petrous to upper cervical segments but with a residual area of irregularity and stenosis more proximally $(A)$. Right ICA after deployment of $5 \times 25-\mathrm{mm}$ PED spanning the upper cervical to the proximal irregular segments $1.8 \mathrm{~cm}$ above the carotid bifurcation, showing restoration of luminal caliber (B). C and D: 3-month (C) and 6-month (D) follow-up MRA sequences demonstrating progressive healing of multiple dissections and good distal flow within the anterior circulation bilaterally.

night change in vessel caliber and was treated with 2 Neuroform Atlas stents, which were approved by the FDA in 2017. The Atlas is a self-expanding, nitinol, laser-cut mini-stent with an open cell design developed for stentassisted aneurysm coiling. The Atlas has several technical advantages over previous models, principally a lower delivery profile. ${ }^{40,44}$ However, it has a relatively low outward radial force, favoring balloon predilatation prior to deployment. ${ }^{8,24}$ Our patient additionally received 2 PEDs for her longer left cervical and petrous ICA dissection. PEDs, also well described in aneurysm management, are flexible mesh devices that can conform to vessels despite high degrees of regional tortuosity, and are well suited for (off-label) treatment of complex dissections particularly with intracranial extension. ${ }^{7}$ However, this device is substantially more thrombogenic than the Atlas stent family, and therefore greater attention to the antiplatelet regimen is required. ${ }^{19}$

It is possible for patients with a critical ICA stenosis to initially present without neurological deficits in the setting of robust collateral circulation, which includes, in general descending order of efficiency, the circle of Willis, multiple ECA-ICA anastomoses (principally via the OphA), 

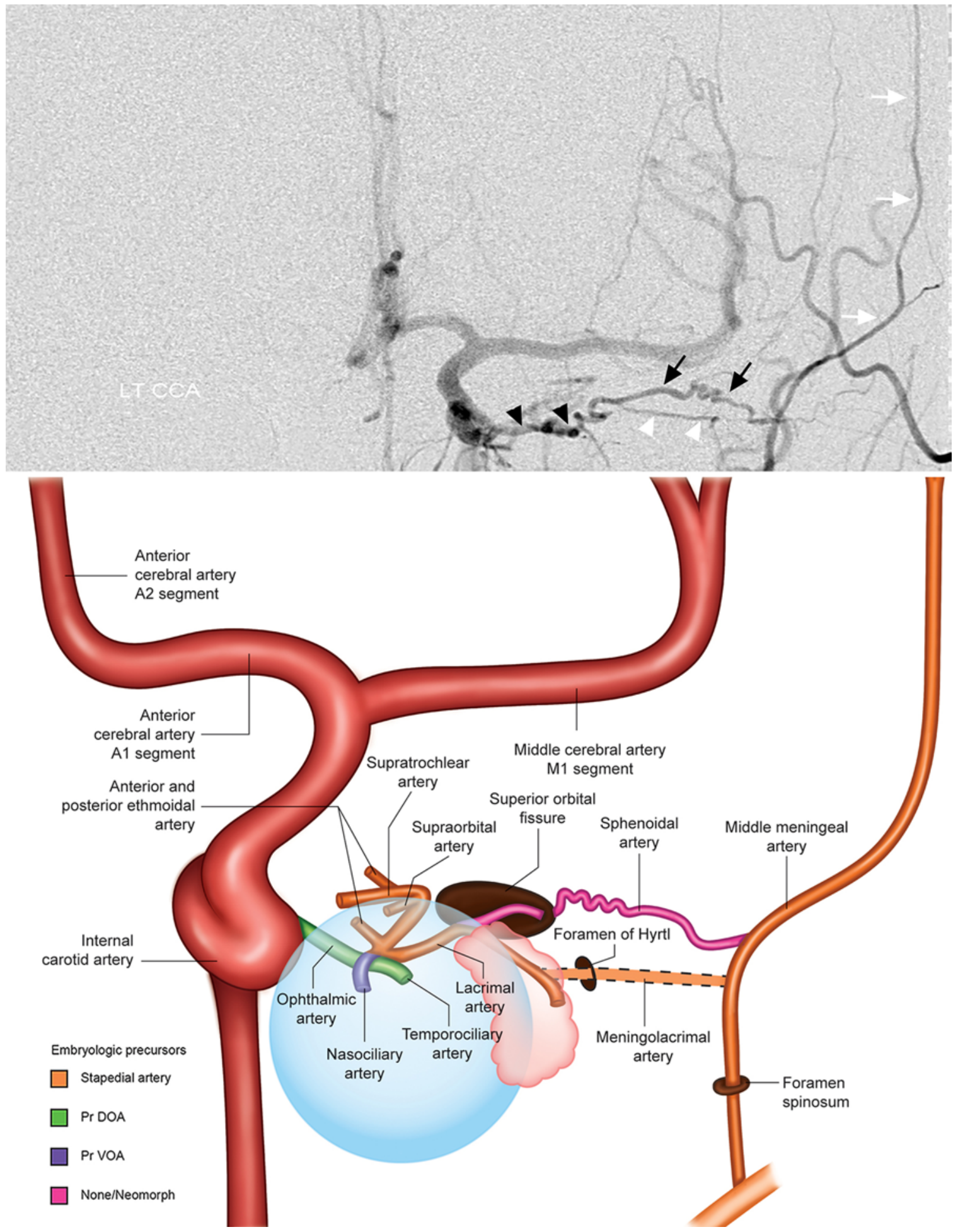

FIG. 5. Upper: Magnified image from interventional angiogram (Fig. 2F) showing early reconstitution of left anterior circulation from MMA (white arrows) to the lacrimal artery (white arrowheads) and OphA (black arrowheads) via a fetal neomorphic remnant, the sphenoidal artery (black arrows). Lower: Anatomical illustration in the anterior view of potential MMA-OphA anastomoses-including the sphenoidal and meningolacrimal arteries_and their associated embryologic origins. Pr DOA = primitive dorsal OphA; Pr VOA = primitive ventral OphA. 
and leptomeningeal collateral vessels. Reconstitution of the OphA can proceed via multiple collaterals, which include the middle meningeal artery (MMA) and accessory meningeal, superficial temporal, deep temporal, infraorbital, and facial arteries. ${ }^{22,29,35}$ In the context of transarterial embolization, it is critical to identify these anastomoses to prevent unintended embolization into the OphA territory. . $^{17,18,27,39}$

In our case, the patient's anastomosis served a protective role: a robust ECA-ICA anastomosis allowed for sufficient collateral flow to her dominant hemisphere despite a $>90 \%$ left ICA stenosis and limited efficiency of the circle of Willis and leptomeningeal collaterals due to dissections in the contralateral carotid and VA systems. The principal ICA reconstitution in this case proceeded via an MMA-OphA connection along the greater sphenoid wing, occasionally named the sphenoidal artery (Fig. 5). ${ }^{17}$ The sphenoidal artery enters the superior orbital fissure to anastomose with the proximal OphA. ${ }^{34}$ The full persistence of this anastomosis-with complete MMA supply of the orbit and no identifiable origin of the OphA from the ICA-represents the meningo-ophthalmic variant. ${ }^{18}$ The same sphenoidal artery also participates in more limited MMA supply of the lacrimal gland, known as the meningo-lacrimal artery variant, recognized as a short, straight vessel entering the orbit through its own foramen of Hyrtl. ${ }^{17,32}$ Review of the relevant ontogenetics suggests that the sphenoidal artery may be a vestige of a later-developing neomorph. ${ }^{14}$ This embryological phenomenon is of particular interest to interventionalists because variants involving partial or complete OphA origin of the MMA are more heavily associated with the presence of a sphenoidal artery. ${ }^{14,15}$ This sphenoidal artery variant complicates surgical planning for cases in which an MMA deriving its circulation from the OphA is supplying pathologic processes such as meningiomas, dural arteriovenous fistulas, moyamoya disease, aneurysms, and chronic subdural hematomas. ${ }^{17,46}$

\section{Conclusions}

In SCAD, clinical presentation can be misleading. The presence of robust fetal anastomoses between ECA and ICA circulations can mask the severity of disease and is only visualized on high-resolution intravascular imaging. Identification of potentially dangerous ECA-ICA anastomoses is critical to ensuring safe embolization procedures; however, the same anastomoses can be useful in maintaining cerebral perfusion. Off-label use of the PED and the Atlas stent for revascularization of cervical dissections deserves further study.

\section{Acknowledgments}

Employees at the NYU Radiology 3D Imaging Lab; NYU School of Medicine: Dr. Aaron Lord, Dr. Eytan Raz, Dr. Miguel Litao, Dr. Breehan Chancellor, and Dr. Asya Wallach.

\section{References}

1. Abe A, Nito C, Sakamoto Y, Nogami A, Hokama H, Takahashi S, et al: Spontaneous bilateral cervical internal carotid and vertebral artery dissection in a Japanese patient without collagen vascular disease with special reference to singlenucleotide polymorphisms. J Stroke Cerebrovasc Dis 25:e114-e117, 2016

2. Arnold M, Cumurciuc R, Stapf C, Favrole P, Berthet K, Bousser MG: Pain as the only symptom of cervical artery dissection. J Neurol Neurosurg Psychiatry 77:1021-1024, 2006

3. Biondi A, Katz JM, Vallabh J, Segal AZ, Gobin YP: Progressive symptomatic carotid dissection treated with multiple stents. Stroke 36:e80-e82, 2005

4. Brandt T, Grond-Ginsbach C: Spontaneous cervical artery dissection: from risk factors toward pathogenesis. Stroke 33:657-658, 2002

5. Brandt T, Hausser I, Orberk E, Grau A, Hartschuh W, AntonLamprecht I, et al: Ultrastructural connective tissue abnormalities in patients with spontaneous cervicocerebral artery dissections. Ann Neurol 44:281-285, 1998

6. Brandt T, Orberk E, Weber R, Werner I, Busse O, Müller BT, et al: Pathogenesis of cervical artery dissections: association with connective tissue abnormalities. Neurology 57:24-30, 2001

7. Brzezicki G, Rivet DJ, Reavey-Cantwell J: Pipeline Embolization Device for treatment of high cervical and skull base carotid artery dissections: clinical case series. J Neurointerv Surg 8:722-728, 2016

8. Cho SH, Jo WI, Jo YE, Yang KH, Park JC, Lee DH: Benchtop comparison of physical properties of 4 commerciallyavailable self-expanding intracranial stents. Neurointervention 12:31-39, 2017

9. Chowdhury MM, Sabbagh CN, Jackson D, Coughlin PA, Ghosh J: Antithrombotic treatment for acute extracranial carotid artery dissections: a meta-analysis. Eur J Vasc Endovasc Surg 50:148-156, 2015

10. Debette S, Kamatani Y, Metso TM, Kloss M, Chauhan G, Engelter ST, et al: Common variation in PHACTR1 is associated with susceptibility to cervical artery dissection. Nat Genet 47:78-83, 2015

11. Debette S, Leys D: Cervical-artery dissections: predisposing factors, diagnosis, and outcome. Lancet Neurol 8:668-678, 2009

12. Debette S, Markus HS: The genetics of cervical artery dissection: a systematic review. Stroke 40:e459-e466, 2009

13. Debette S, Metso TM, Pezzini A, Engelter ST, Leys D, Lyrer $\mathrm{P}$, et al: CADISP-genetics: an international project searching for genetic risk factors of cervical artery dissections. Int J Stroke 4:224-230, 2009

14. Diamond MK: Homologies of the meningeal-orbital arteries of humans: a reappraisal. J Anat 178:223-241, 1991

15. Diamond MK: Homologies of the stapedial artery in humans, with a reconstruction of the primitive stapedial artery configuration of euprimates. Am J Phys Anthropol 84:433-462, 1991

16. Dreier JP, Lurtzing F, Kappmeier M, Bohner G, Klingebiel R, Leistner S, et al: Delayed occlusion after internal carotid artery dissection under heparin. Cerebrovasc Dis 18:296-303, 2004

17. Gailloud P, Gregg L, Ruiz DS: Developmental anatomy, angiography, and clinical implications of orbital arterial variations involving the stapedial artery. Neuroimaging Clin $\mathbf{N}$ Am 19:169-179, 2009

18. Geibprasert S, Pongpech S, Armstrong D, Krings T: Dangerous extracranial-intracranial anastomoses and supply to the cranial nerves: vessels the neurointerventionalist needs to know. AJNR Am J Neuroradiol 30:1459-1468, 2009

19. Girdhar G, Li J, Kostousov L, Wainwright J, Chandler WL: In-vitro thrombogenicity assessment of flow diversion and aneurysm bridging devices. J Thromb Thrombolysis 40:437-443, 2015

20. Grond-Ginsbach C, Chen B, Pjontek R, Wiest T, Jiang Y, 
Burwinkel B, et al: Copy number variation in patients with cervical artery dissection. Eur J Hum Genet 20:1295-1299, 2012

21. Hassan AE, Zacharatos H, Mohammad YM, Tariq N, Vazquez G, Rodriguez GJ, et al: Comparison of single versus multiple spontaneous extra- and/or intracranial arterial dissection. J Stroke Cerebrovasc Dis 22:42-48, 2013

22. Hayreh SS: The ophthalmic artery: III. Branches. Br J Ophthalmol 46:212-247, 1962

23. Kloss M, Wiest T, Hyrenbach S, Werner I, Arnold ML, Lichy C, et al: MTHFR 677TT genotype increases the risk for cervical artery dissections. J Neurol Neurosurg Psychiatry 77:951-952, 2006

24. Krischek O, Miloslavski E, Fischer S, Shrivastava S, Henkes $\mathrm{H}$ : A comparison of functional and physical properties of self-expanding intracranial stents [Neuroform3, Wingspan, Solitaire, Leo+, Enterprise]. Minim Invasive Neurosurg 54:21-28, 2011

25. Kuhlenbäumer G, Friedrichs F, Kis B, Berlit P, Maintz D, Nassenstein I, et al: Association between single nucleotide polymorphisms in the lysyl oxidase-like 1 gene and spontaneous cervical artery dissection. Cerebrovasc Dis 24:343348,2007

26. Lee VH, Brown RD Jr, Mandrekar JN, Mokri B: Incidence and outcome of cervical artery dissection: a population-based study. Neurology 67:1809-1812, 2006

27. Loon NW, Gendeh BS, Zakaria R, Hamzah JC, Din NM: Ophthalmic artery occlusion following neuro-embolization of the external carotid artery, a case report. BMC Ophthalmol 17:92, 2017

28. Loret JE, Francois P, Papagiannaki C, Cottier JP, Terrier LM, Zemmoura I: Internal carotid artery dissection after anterior cervical disc replacement: first case report and literature review of vascular complications of the approach. Eur J Orthop Surg Traumatol 23 (Suppl 1):S107-S110, 2013

29. Macchi V, Regoli M, Bracco S, Nicoletti C, Morra A, Porzionato A, et al: Clinical anatomy of the orbitomeningeal foramina: variational anatomy of the canals connecting the orbit with the cranial cavity. Surg Radiol Anat 38:165-177, 2016

30. Markus HS, Hayter E, Levi C, Feldman A, Venables G, Norris $\mathrm{J}$ : Antiplatelet treatment compared with anticoagulation treatment for cervical artery dissection (CADISS): a randomised trial. Lancet Neurol 14:361-367, 2015

31. Michel P, Odier C, Rutgers M, Reichhart M, Maeder P, Meuli $\mathrm{R}$, et al: The Acute STroke Registry and Analysis of Lausanne (ASTRAL): design and baseline analysis of an ischemic stroke registry including acute multimodal imaging. Stroke 41:2491-2498, 2010

32. Moret J, Lasjaunias P, Théron J, Merland JJ: The middle meningeal artery. Its contribution to the vascularisation of the orbit. J Neuroradiol 4:225-248, 1977

33. Müller BT, Luther B, Hort W, Neumann-Haefelin T, Aulich A, Sandmann W: Surgical treatment of 50 carotid dissections: indications and results. J Vasc Surg 31:980-988, 2000

34. Padget D: The development of the cranial arteries in the human embryo. Contrib Embryol 32:205-261, 1948

35. Perrini P, Cardia A, Fraser K, Lanzino G: A microsurgical study of the anatomy and course of the ophthalmic artery and its possibly dangerous anastomoses. J Neurosurg 106:142150,2007
36. Rubinstein SM, Peerdeman SM, van Tulder MW, Riphagen I, Haldeman S: A systematic review of the risk factors for cervical artery dissection. Stroke 36:1575-1580, 2005

37. Schievink WI: Spontaneous dissection of the carotid and vertebral arteries. N Engl J Med 344:898-906, 2001

38. Silbert PL, Mokri B, Schievink WI: Headache and neck pain in spontaneous internal carotid and vertebral artery dissections. Neurology 45:1517-1522, 1995

39. Soong HK, Newman SA, Kumar AA: Branch artery occlusion. An unusual complication of external carotid embolization. Arch Ophthalmol 100:1909-1911, 1982

40. Ten Brinck MFM, de Vries J, Bartels RHMA, Grotenhuis JA, Boogaarts HD: NeuroForm Atlas stent-assisted coiling: preliminary results. Neurosurgery [epub ahead of print], 2018

41. Townend BS, Traves L, Crimmins D: Bilateral spontaneous carotid artery dissection. J Clin Neurosci 12:592-594, 2005

42. Tsivgoulis G, Safouris A, Alexandrov AV: Safety of intravenous thrombolysis for acute ischemic stroke in specific conditions. Expert Opin Drug Saf 14:845-864, 2015

43. Ulbricht D, Diederich NJ, Hermanns-Lê T, Metz RJ, Macian F, Piérard GE: Cervical artery dissection: an atypical presentation with Ehlers-Danlos-like collagen pathology? Neurology 63:1708-1710, 2004

44. Ulfert C, Pham M, Sonnberger M, Amaya F, Trenkler J, Bendszus M, et al: The Neuroform Atlas stent to assist coil embolization of intracranial aneurysms: a multicentre experience. J Neurointerv Surg 10:1192-1196, 2018

45. Xianjun $\mathrm{H}$, Zhiming Z: A systematic review of endovascular management of internal carotid artery dissections. Intervent Neurol 1:164-170, 2013

46. Yu J, Guo Y, Xu B, Xu K: Clinical importance of the middle meningeal artery: a review of the literature. Int J Med Sci 13:790-799, 2016

\section{Disclosures}

Dr. Maksim Shapiro discloses a consultant and Pipeline device proctor relationship with Medtronic, Inc.

\section{Author Contributions}

Conception and design: Golub, Torres, Shapiro. Acquisition of data: Golub, Hu. Analysis and interpretation of data: Golub, Torres. Drafting the article: Golub, $\mathrm{Hu}$, Dogra. Critically revising the article: all authors. Reviewed submitted version of manuscript: all authors. Approved the final version of the manuscript on behalf of all authors: Golub. Administrative/technical/material support: Golub, Dogra, Torres, Shapiro. Study supervision: Golub, Torres, Shapiro.

\section{Correspondence}

Danielle Golub: NYU School of Medicine, New York, NY. danielle.golub@nyumc.org. 